\author{
Alexandre Fortier \\ Elaine Ménard \\ McGill University, Montréal, Québec, Canada
}

\title{
Communication : Quelles données satisferaient les utilisateurs de sites web de musées?
}

\begin{abstract}
This project examines the fundamental elements for the description of museum objects and their modelization using linked open data. In this phase, the needs and expectations of 60 museum website users regarding the descriptive data associated with museum objects of any kind on museum websites are presented.

Résumé : Ce projet propose d'examiner les éléments essentiels à la description des objets muséaux et modéliser ceux-ci à l'aide de données ouvertes liées. Dans cette phase, les attentes et les besoins d'un groupe de 60 utilisateurs relativement aux données descriptives associées aux objets muséaux de toutes natures sur les sites web de musées sont présentés.
\end{abstract}

\section{Introduction}

Les institutions culturelles et patrimoniales telles que les musées se doivent de fournir au public un accès virtuel à leurs collections. Toutefois, cet accès est souvent limité par de nombreux facteurs, dont l'incompatibilité des données et leur mode de repérage. En effet, les musées travaillent souvent en vase clos, n'utilisant pas les mêmes méthodes de description ou d'accès. Ce manque d'interopérabilité a pour conséquence la quasiimpossibilité d'échanger des informations d'un musée à un autre, décuplant ainsi la tâche de chacun d'eux. En outre, les musées doivent souvent se contenter d'offrir à leurs usagers des descriptions limitées à quelques informations textuelles pour décrire leurs objets, souvent choisies pour des besoins internes et non pas pour être diffusées au grand public. L'utilisation de données liées ouvertes (linked open data) semble donc faire partie de la solution pour pallier le manque manifeste de compatibilité et pour changer la manière avec laquelle l'utilisateur interagit avec l'information.

L'idée derrière l'utilisation d'une infrastructure web pour rendre les métadonnées accessibles et améliorer leur interopérabilité n'est pas nouvelle. Des projets utilisant les données ouvertes liées se sont spécifiquement intéressés aux objets muséaux: la bibliothèque virtuelle Europeana (Europeana Labs 2016), le projet Amsterdam Museum Linked Open Data (de Boer et al. 2012), le projet du Smithsonian American Art Museum (Szekely et al. 2013) et le projet du Linked Open Data for Academia (Kamura et al. 2011), notamment. Au Canada, certains projets pilotes ont été lancés, comme «Au-delà des tranchées » du Réseau pancanadien du patrimoine documentaire (2015). Ces projets ne sont toutefois pas encore répandus et plusieurs musées hésitent toujours à aller dans cette direction. Les musées apparaissent aussi plus souvent plus préoccupés par la numérisation des données sur les objets et leur mise en ligne que par leur utilisation. Les attentes des utilisateurs de sites web de musées demeurant plutôt méconnues. 


\section{Objectifs}

Cette communication présente les attentes et les besoins d'un groupe d'utilisateurs relativement aux données descriptives associées aux objets muséaux de toutes natures sur les sites web de musées. Elle s'intéresse également à la manière avec laquelle les données ouvertes liées peuvent enrichir leur expérience.

Cette communication s'inscrit dans le cadre d'un plus vaste projet de recherche qui propose d'examiner les éléments essentiels à la description des objets muséaux et modéliser ceux-ci à l'aide de données ouvertes liées. Plus spécifiquement, ce projet cherche à remplir trois objectifs : (1) comprendre les caractéristiques nécessaires à la description des objets muséaux de toute nature ; (2) définir un modèle pour la description des objets muséaux à l'aide des données ouvertes liées; et (3) renforcer les réseaux et échanges de données entre diverses institutions culturelles et patrimoniales.

\section{Méthodologie}

Différentes méthodologies ont été proposées et testées pour inclure les utilisateurs dans le processus création d'interfaces et de systèmes d'information. Parmi celles-ci, l'Informant Design requiert de recevoir les commentaires des utilisateurs à plusieurs stages du processus de création; les participants étant considérés comme des informateurs qui peuvent aider les chercheurs à des stades clés de leur processus (Scaife et Rogers 1999). Ainsi, pour cette partie du projet de recherche, les données ont été collectées par le biais d'un échantillon à choix raisonné (Patton 2015) constitué de 60 utilisateurs de sites web de musées. Les participants ont d'abord rempli un questionnaire comprenant des questions fermées et ouvertes sur leurs habitudes de fréquentation sur les sites web de musées et leurs attentes reliées aux objets présentés sur ces sites. Ils ont ensuite participé à une entrevue individuelle. Les données recueillies ont été examinées par une analyse de contenu basée sur la théorisation ancrée, dont le but est de développer ou de raffiner, à l'aide des thèmes, catégories et patrons observés, une théorie (Corbin et Strauss 1990; 2015; Glaser et Strauss 1967). La théorisation ancrée utilise un codage hiérarchique et ouvert qui permet l'ajout de nouvelles catégories au fur et à mesure que celles-ci sont identifiées, une caractéristique essentielle lorsque, comme pour ce projet, le guide de codification doit être enrichi progressivement grâce aux données recueillies dans les entrevues (Corbin et Strauss 2015; Patton 2015). Les différents thèmes émergents des données ont été codés et utilisés de manière itérative au cours de l'analyse. Afin d'assurer la validité et la fiabilité de l'analyse, une évaluation intercodeur a été réalisée pour évaluer la fiabilité du système de codage développé par les chercheurs. Les taux d'accord indiquent que le schéma de codage était compréhensible et reflète les thèmes révélés par les données.

\section{Résultats}

L'analyse préliminaire des résultats indique que les utilisateurs de sites web de musées trouvent que les musées, même parmi les institutions les plus importantes, présentent peu d'informations intéressantes sur les objets dans leurs collections. Les métadonnées descriptives (par exemple, les dimensions, et les matériaux ou le médium) qui forment la majorité des descriptions d'objets muséaux sont perçues comme inintéressantes. De plus amples informations à propos de l'origine culturelle ou géographique ainsi que sur la 
période de laquelle un objet est issu sont de plus grand intérêt. Les participants expriment également leur désir d'en savoir davantage sur le créateur de l'objet et sur une mise en contexte. De nombreux participants désireraient que les sites web de musées leur en apprennent plus sur les objets à la manière d'un guide érudit qui, par sa connaissance, leur redonne vie lors d'une visite guidée.

La connaissance des données ouvertes liées est très basse, voire inexistante, parmi les participants. Par contre, lorsque questionnés sur les possibilités offertes par les données ouvertes liées, notamment la découverte d'objets similaires dans la collection ou dans une autre collection, les participants démontrent un grand intérêt pour ce type de découvertes.

\section{Conclusion}

L'utilisation de données ouvertes liées permet le décloisonnement des institutions culturelles. Pouvoir consulter simultanément et aisément plusieurs bases de données aura pour résultat une mise en contexte sans précédent de l'objet muséal. La description de l'objet sera étoffée non seulement par les données contenues dans la base propre d'un musée, mais aussi par des éléments tirés d'autres bases de données, et, cela, sans frontière linguistique puisque les éléments mis en réseau peuvent avoir été conçus en multiples langues. Pour satisfaire leurs utilisateurs, par contre, les sites web de musées doivent songer à la qualité de l'information présentée et non au simple volume de notices d'objets numérisées. Le site web et le musée doivent être vus comme étant complémentaires. Un site web de musées ne doit pas simplement inciter les gens à venir en personne, mais aussi permettre d'en connaître davantage sur ce que l'on a vu pendant cette visite. L'élaboration d'un modèle pour la description des objets muséaux à l'aide des données ouvertes liées doit en tenir compte pour maximiser son impact auprès de ceux qui visitent le site web.

\section{Références}

Corbin, J. et Strauss, A. (1990). Grounded theory research: procedures, canons, and evaluative criteria. Qualitative Sociology, 13(1), 3-21.

Corbin, J. et Strauss, A. (2015). Basics of qualitative research: Techniques and procedures for developing grounded theory ( $4^{\mathrm{e}}$ éd.). Thousand Oaks, Calif. : Sage.

De Boer, V., Wielemaker, J., Gent, J., Hildebrand, M., Isaac, A., Ossenbruggen, J. et Schreiber, G. 2012. Supporting linked data production for cultural heritage institutes: The Amsterdam Museum case study. Dans E. Simperl, P. Cimiano, A. Polleres, O. Corcho et V. Presutti (dir.), The semantic web: Semantics and big data (p. 733-747). Berlin : Springer.

Europeana Labs (2016) Europeana Linked Open Data. Repéré : http://labs.europeana.eu/api/linked-open-data/introduction/.

Glaser, B. et Strauss, A. (1967). The discovery of grounded theory: Strategies for qualitative research. Chicago : Aldine de Gruyter. 
Kamura, T., Takeda, H., Ohmukai, I., Kato, F., Takahashi, T. et Ueda, H. 2011. Study support and integration of cultural information resources with linked data. Dans Proceedings of the 2011 Second International Conference on Culture and Computing (p. 177-178). Washington, D.C. : IEEE Computer Society.

Patton, M. Q. (2015). Qualitative research \& evaluation methods: Integrating theory and practice ( $4^{\mathrm{e}}$ éd.). Thousand Oaks, Calif. : Sage.

Réseau pancanadien du patrimoine documentaire. 2015. Démonstration de faisabilité de la visualisation des données ouvertes liées (LOD). Repéré : http://www.canadiana.ca/ sites/pub.canadiana.ca/files/PCDHN\%20Proof-ofconcept_Final-Report-FRA_0.pdf.

Scaife, M. et Rogers, Y. (1999). Kids as informants: Telling us what we didn't know or confirming what we knew already, dans A. Druin (dir.), The design of children's technology (p. 27-50). San Francisco : Kaufmann.

Szekely, P., Knoblock, C. A., Yang, F., Zhu, X., Fink, E. E., Allen, R. et Goodlander, G. (2013). Connecting the Smithsonian American Art Museum to the linked data cloud. Dans E. Simperl, P. Cimiano, A. Polleres, O. Corcho et V. Presutti (dir.), The semantic web : Semantics and big data (p.593-607). Berlin : Springer. 